\title{
A control rule for planning promotion in a Nigerian university setting
}

\author{
Virtue U. Ekhosuehi ${ }^{1, \dagger}$ \\ ${ }^{1}$ Department of Mathematics, University of Benin, P.M.B. 1154, Benin City, Nigeria \\ E-mail: 〈virtue.ekhosuehi@uniben.edu〉
}

\begin{abstract}
This paper examines the attainability problem in a graded manpower system, where the objective is to maximise the total throughput. The problem is modelled as a linear programming problem (LPP) and the evolution of structures in the system is described using the Markov chain model. The decision variables are the promotion rates. Results from the LPP provide a guide to the administrative authority of the system on how promotion and retrenchment should be implemented. The utility of the model is demonstrated using a university setting in Nigeria.
\end{abstract}

Keywords: attainability, linear programming, manpower system, Markov chain, promotion control

Received: July 9, 2015; accepted: September 7, 2016; available online: December 30, 2016

DOI: $10.17535 /$ crorr. 2016.0012

\section{Introduction}

This paper concentrates on the attainability problem under promotion control, where retrenchment is allowed. The paper considers a manpower system stratified into $k$ categories according to the grades. Let $S$ be the set of categories and let each staff belong to one and only one category in $S$. Let the number of staff in each category of the system at a period of time $t$ be denoted by the $k$-tuple stock vector $\mathbf{x}(t)=\left[x_{1}(t), \cdots, x_{k}(t)\right]$. The vector $\mathbf{x}(t)$ is also referred to as the structure of the system at time $t$. The stock $x_{i}(t)$ changes after a period of time in such fashion that: a proportion $p_{i i}$ is still in category $i$, a proportion $p_{i j}$ is promoted to the next higher category $i+1, i, j=i+1 \in S$, a proportion $p_{i 0}$ leaves the system in category $i, 0 \notin S$, or a proportion of recruits, $r_{i}$, enter category $i$. The problem of attaining a desired structure $\mathbf{x}^{*}$ with the structure $\mathbf{x}$ as the starting point involves finding either the transition matrix $\mathbf{P}=\left(p_{i j}\right)_{i, j \in S}$ or the recruitment distribution $\mathbf{r}$, such that a structure $\widetilde{\mathbf{x}}$ is attained which is

$\dagger$ Corresponding author 
as close as possible to $\mathbf{x} *$ [4]. When the task is to find $\mathbf{r}$, the strategy is called recruitment control; otherwise, it is called promotion control. In either case, one of the policy matrices is given. Works on recruitment control are popular in the literature, whereas little has been said about promotion control [4, 5, 9]. Nilakantan [21] considered a manpower system which operates under certain recruitment restrictions as a means to protect the career prospects of its members. Later on, Nilakantan [22] evaluated the manpower system which seeks to control the blend of internal and outsource manpower. Udom [29] studied manpower control for a hierarchical system based on promotion and interdepartmental transfers. The study assumed that several policy matrices are available and that the task of the manpower planner was to determine the policy matrix that will minimise the total cost. The present study relaxes this assumption and seeks to determine the policy matrix that maximises the throughput per staff.

This study was originally motivated by an examination of the model for stationarity with promotion control [4]. The model was constructed on the assumption that recruitment is done to replace wastage and to achieve the desired growth at a rate of expansion, $\alpha$. The model for the attainability problem under promotion control is expressed as

$$
\mathbf{q}(t) \mathbf{P}=(1+\alpha) \mathbf{D}-\mathbf{q}(t)\left(\mathbf{w}^{\prime}+\mathbf{e}^{\prime} \alpha\right) \mathbf{r}
$$

where $\mathbf{q}(t)$ is the relative structure of the system at time $t, \mathbf{W}$ is a $1 \times k$ vector of wastage probabilities, and $\mathbf{r}$ is a $1 \times k$ vector of the distribution of recruits. The prime is used to denote a matrix-vector transposition. The distribution of the attainable structure for the system is given by the $1 \times k$ vector, $\mathbf{D}=\left(d_{i}\right)$. The vector $\mathbf{D}$ is such that $\mathbf{D e}=1$, where $\mathbf{e}$ is a $k \times 1$ vector of ones. As at the time of writing this paper, the only promotion control model in discrete-time known to the author that is similar to this study design is that of Bartholomew et al. [4]. Mathematical models for manpower planning abound in the literature $[2,3,4,11$, $12,14,24,30,33]$. Among these models is the Markov chain model, which is commonly used for systems with countable state space $[10,13,17,18,27,28,31$, 32]. The Markov chain model provides a means of unifying the states of the manpower system. More so, the Markov models have been used as a theoretical framework to assess the underlying nature of the flows of students through educational systems $[8,19,20]$.

This study complements the existing literature on the mathematics of manpower planning by focusing on the aspect of promotion control. The study attempts to answer the following questions: 'Given that the administrative authority of a manpower system is considering recruiting a certain number of staff such that the staff structure closely follows a certain attainability requirement, what then should be the promotion rates in the system in order to achieve the requirement? Will this lead to retrenchment? If it does, how many staff should be retrenched?' 
The study assumes that transitions in the manpower system take place at discrete times $[4,16]$ and that the objective is to maximise the throughput per staff [23]. Another assumption is that the recruitment is carried out gradually so much so that the new recruits in the system at any given period do not exceed the existing staff strength. For convenience, the recruitment is assumed to take place at the end of the period according to the distribution $\mathbf{r}=\left[r_{1}, \cdots, r_{k}\right]$. The attainability problem of the manpower system is formulated as a linear programming problem [15] within the Markov chain framework. The basic idea of this study is to find the one-step transition matrix $\mathbf{P}=\left(p_{i j}\right)_{i, j \in S}$, which maximises the throughput without violating the admissible conditions on the $p_{i j}$

$$
\text { (i.e., } 0 \leq p_{i j} \leq 1 \text { and } \sum_{j=1}^{k} p_{i j} \leq 1 \text { ), }
$$

given the number of new recruits and the attainable structure. The task of finding the one-step transition matrix is a non-trivial problem. This is because of the possibility of attrition of staff in the system. Davis et al. [6] had earlier provided a way to obtain estimates of the one-step transition probabilities from irregularly spaced data based on partial odds. This study adopts the linear programming (LP) approach so as to incorporate the objective function of the system, as well as the constraints. Dantzig's rule is used as the pivoting rule [26]. The structure derived from the solution for the constrained optimization problem is the optimal structure. The results obtained from the model proposed in this study are compared with that obtained using the model of Bartholomew et al. [4]. The optimal structure obtained by our model is denoted as $\mathbf{x}^{\text {opt }}$. Generally, we use the superscript opt to denote the optimal value and the superscript Barth to denote value obtained using the model of Bartholomew et al. [4].

This study highlights: (1) how to find a one-step optimal transition matrix under promotion control for a manpower system; (2) how to determine the specific category in a manpower system where retrenchment should be done whenever it is carried out; (3) the use of throughput as a major goal in the manpower system, rather than utilising the profit-seeking motive and the cost reduction approach $[1,23,29]$; (4) a normative approach based on linear programming as a solution technique to the attainability problem in manpower systems; and (5) the decision making under uncertainty with the aid of the Markov chain framework.

\section{Model description}

The evolution of structures in a manpower system is commonly modelled using the Markov chain framework as [4, 9]:

$$
\mathbf{x}(t+1)=\mathbf{x}(t) \mathbf{P}+h(t+1) \mathbf{r}, \quad t=0,1,2, \cdots
$$


where $h(t+1)$ is the number of new recruits, which, oftentimes, is decided by the administrative authority of the system. In the absence of attrition such as retrenchment, voluntary withdrawal, dismissal, retirement, etc., the desired structure is achieved when

$$
\mathbf{x}(t+1)=(\mathbf{x}(t) \mathbf{e}+h(t+1)) \mathbf{D}
$$

Since wastage occurs in practice, we have

$$
\mathbf{x}(t+1) \leq(\mathbf{x}(t) \mathbf{e}+h(t+1)) \mathbf{D}
$$

Therefore

$$
\mathbf{x}(t) \mathbf{P} \leq \mathbf{b}
$$

where $\mathbf{b}=(\mathbf{x}(t) \mathbf{e}+h(t+1)) \mathbf{D}-h(t+1) \mathbf{r}$. This study assumes that the right hand side (RHS) values of equation (5), i.e., the entries in vector $\mathbf{b}$, are nonnegative. This is because a negative entry would imply that $\mathbf{x}(t) \mathbf{e}<h(t+1)$ for some $d_{i}<r_{i}$. Thus, if any of the RHS values is negative, then it is set equal to zero.

The structure of the transition matrix $\mathbf{P}$ is determined from the promotion policy of the manpower system. For instance, in a 3 -graded manpower system where the policy is that both demotion and double promotion are not allowed, the transition matrix is

$$
\mathbf{P}=\left[\begin{array}{ccc}
p_{11} & p_{12} & 0 \\
0 & p_{22} & p_{23} \\
0 & 0 & p_{33}
\end{array}\right]
$$

The admissible conditions for the matrix $\mathbf{P}=\left(p_{i j}\right)_{i, j \in S}$ are that $\mathbf{P}$ is substochastic and that $p_{i j} \geq 0$ for $i, j \in S$. Considering equation (1) for the 3 graded manpower system, we have

$$
\left[\begin{array}{lll}
q_{1} & q_{2} & q_{3}
\end{array}\right]\left[\begin{array}{ccc}
p_{11} & p_{12} & 0 \\
0 & p_{22} & p_{23} \\
0 & 0 & p_{33}
\end{array}\right]=\left[\begin{array}{lll}
f_{1} & f_{2} & f_{3}
\end{array}\right]
$$


where $q_{i}$ is the $i$ th entry in the vector $\mathbf{q}(t)$ and $f_{i}$ is the $i$ th entry in the vector obtained by simplifying the expression $(1+\alpha) \mathbf{D}-\mathbf{q}(t)\left(\mathbf{w}^{\prime}+\mathbf{e}^{\prime} \alpha\right) \mathbf{r}$. Since the matrix $\mathbf{P}$ is being sought for under promotion control, we equate both sides of equation (7) and get

$$
\begin{array}{r}
p_{11}=\frac{f_{1}}{q_{1}} \\
q_{1} p_{12}+q_{2} p_{22}=f_{2} \\
q_{2} p_{23}+q_{3} p_{33}=f_{3}
\end{array}
$$

There are infinitely many solutions to the simultaneous equations (9) and (10). To ensure that the solutions does not violate the stochastic property that

$$
\sum_{j=1}^{3} p_{i j}+p_{i 0}=1
$$

with $p_{i 0}$ being the $i$ th entry in $\mathbf{w}$, equation (11) is introduced as a constraint. It turns out that the use of this constraint gives

$$
\begin{aligned}
& p_{12}=1-\frac{1}{q_{1}}\left(f_{1}+q_{1} p_{10}\right) \\
& p_{22}=\frac{1}{q_{2}}\left(f_{1}+f_{2}+q_{1} p_{10}-q_{1}\right) \\
& p_{23}=1-\frac{1}{q_{2}}\left(f_{1}+f_{2}+q_{1} p_{10}+q_{2} p_{20}-q_{1}\right)
\end{aligned}
$$

and

$$
p_{33}=\frac{1}{q_{3}}\left(f_{1}+f_{2}+f_{3}+q_{1} p_{10}+q_{2} p_{20}-q_{1}-q_{2}\right)
$$

However, there is no guarantee that $p_{33}$ in equation (15) will be equal to $1-p_{30}$. Thus $p_{33}$ may not be uniquely determined. Another limitation of the model [4] is that it may give a non-admissible solution for $\mathbf{P}$. This is the case whenever 
$\mathbf{q}(t) \mathbf{P}<(1+\alpha) \mathbf{D}-\mathbf{q}(t)\left(\mathbf{w}^{\prime}+\mathbf{e}^{\prime} \alpha\right) \mathbf{r}$ for at least one element in the matrix-vector product. These limitations became a driving force to consider an alternative formulation for the attainability problem under promotion control. More specifically, the problem is formulated as a linear programming problem within the Markov chain framework with a view to circumventing the possibility of obtaining a non-admissible solution and uniquely determining the promotion rates. This new formulation is a more advanced model than the one in the literature [4].

Let $\Pi=\left(\pi_{i}\right)$ be a $k \times 1$ vector of the throughput per employee and $z$ the total throughput. Then, the objective function for the manpower system is specified as: $\max z=\mathbf{x}(t) \mathbf{P} \Pi$. Notice that the new recruits $h(t+1)$ are not included. This is because new recruits are treated as if they all came into the system at the end of the period. Thus, the attainability problem is formulated as a linear programming problem (LPP) of the form

\section{LPP 1:}

subject to$$
\max \quad z=\mathbf{x}(t) \mathbf{P} \Pi
$$

$$
\begin{gathered}
\mathbf{x}(t) \mathbf{P} \leq \mathbf{b} \\
\mathbf{P e} \leq \mathbf{e} \\
p_{i j} \geq 0 \text { for } i, j \in S .
\end{gathered}
$$

Rearranging LPP 1 gives

\section{LPP 2:}

$$
\max z=\Phi \mathbf{Y}
$$

subject to

$$
\mathbf{A Y} \leq \Delta
$$

where $\Phi$ is a row vector containing the product $x_{i}(t) \pi_{j} ; \mathbf{A}$ is a matrix of the functional constraints with entries 0,1 and $x_{i}(t) ; \mathbf{Y}$ is a column vector of the $p_{i j}$; and $\Delta$ is a column vector made up of the transpose of vector $\mathbf{b}$ and 1. Returning to the policy matrix of the 3 -graded system in equation (6), we have

$$
\Phi=\left[x_{1}(t) \pi_{1} \quad x_{1}(t) \pi_{2} \quad x_{2}(t) \pi_{2} \quad x_{2}(t) \pi_{3} \quad x_{3}(t) \pi_{3}\right],
$$




$$
\mathbf{A}=\left[\begin{array}{ccccc}
x_{1}(t) & 0 & 0 & 0 & 0 \\
0 & x_{1}(t) & x_{2}(t) & 0 & 0 \\
0 & 0 & 0 & x_{2}(t) & x_{3}(t) \\
1 & 1 & 0 & 0 & 0 \\
0 & 0 & 1 & 1 & 0 \\
0 & 0 & 0 & 0 & 1
\end{array}\right], \mathbf{Y}=\left[\begin{array}{c}
p_{11} \\
p_{12} \\
p_{22} \\
p_{23} \\
p_{33}
\end{array}\right] \text { and } \Delta=\left[\begin{array}{c}
b_{1} \\
b_{2} \\
b_{3} \\
1 \\
1 \\
1
\end{array}\right]
$$

The solution technique to LPP 2 involves introducing a $2 k \times 1$ column vector of slack variables, $\mathbf{Y}_{s}$, so that the problem becomes

$$
\begin{array}{r}
z-\Phi \mathbf{Y}=0 \\
{[\mathbf{A}, \mathbf{I}]\left[\begin{array}{c}
\mathbf{Y} \\
\mathbf{Y}_{s}
\end{array}\right]=\Delta}
\end{array}
$$

or, equivalently,

$$
\left[\begin{array}{ccc}
1 & -\Phi & \mathbf{0}^{\prime} \\
\mathbf{0} & \mathbf{A} & \mathbf{I}
\end{array}\right]\left[\begin{array}{c}
Z \\
\mathbf{Y} \\
\mathbf{Y}_{s}
\end{array}\right]=\left[\begin{array}{l}
0 \\
\Delta
\end{array}\right]
$$

where $\mathbf{0}$ is a $2 k \times 1$ null vector and $\mathbf{I}$ is a $2 k \times 2 k$ identity matrix. The entries in $\mathbf{Y}_{s}$ are the initial basic variables with the inverse basis matrix $\mathbf{B}_{(0)}^{-1}=\mathbf{I}$. Thereafter, the inverse basis matrix $\mathbf{B}_{(v)}^{-1}$ for the $v$ th iteration as well as the simplex multiplier $\Phi_{\mathbf{B}_{v}} \mathbf{B}_{(v)}^{-1}$ is determined using the Dantzig's rule $[15,26] . \Phi_{\mathbf{B}_{v}}$ is the coefficient of the initial non-basic variables of the objective function at the $v$ th iteration. The new set of equations at any iteration is obtained by evaluating

$$
\left[\begin{array}{cc}
1 & \Phi_{\mathbf{B}_{v}} \mathbf{B}_{(v)}^{-1} \\
\mathbf{0} & \mathbf{B}_{(v)}^{-1}
\end{array}\right]\left[\begin{array}{ccc}
1 & -\Phi & \mathbf{0}^{\prime} \\
\mathbf{0} & \mathbf{A} & \mathbf{I}
\end{array}\right]\left[\begin{array}{c}
Z \\
\mathbf{Y} \\
\mathbf{Y}_{s}
\end{array}\right]=\left[\begin{array}{cc}
1 & \Phi_{\mathbf{B}_{v}} \mathbf{B}_{(v)}^{-1} \\
\mathbf{0} & \mathbf{B}_{(v)}^{-1}
\end{array}\right]\left[\begin{array}{l}
0 \\
\Delta
\end{array}\right]
$$

The iteration continues until the difference $\Phi_{\mathbf{B}_{v}} \mathbf{B}_{(v)}^{-1} \mathbf{A}-\Phi$ contains only nonnegative entries. This is the stopping rule. The entries in $\mathbf{Y}$ at which the stopping 
rule is attained are referred to as the optimal solution. Let $\mathbf{Y}^{\text {opt }}$ denote the optimal solution. The vector $\mathbf{Y}^{\text {opt }}$ is used to construct the optimal transition matrix. For instance, in the 3 -graded system, $y_{1}^{\text {opt }}=p_{11}^{\text {opt }}, \quad y_{2}^{\text {opt }}=p_{12}^{\text {opt }}$, $y_{3}^{\text {opt }}=p_{22}^{\text {opt }}, y_{4}^{\text {opt }}=p_{23}^{\text {opt }}$ and $y_{5}^{\text {opt }}=p_{33}^{\text {opt }}$. Let $\mathbf{P}^{\text {opt }}$ be the optimal transition matrix formed from $\mathbf{Y}^{\text {opt }}$. Then the optimal structure at $t+1$ is

$$
\mathbf{x}^{o p t}(t+1)=\mathbf{x}(t) \mathbf{P}^{o p t}+h(t+1) \mathbf{r}
$$

When $p_{i i}^{\text {opt }}=1$, the optimal policy is that the staff in category $i$ should be stagnated. On the other hand, when $0 \leq p_{i i}^{\text {opt }}<1$, the optimal policy recommends that a proportion of $\left(1-p_{i i}^{\text {opt }}\right)$ staff should leave category $i$. The extent to which $\mathbf{x}^{\text {opt }}(t+1)$ closely follows the desired distribution is determined using the Euclidean norm:

$$
E=\left|\mathbf{x}^{o p t}(t+1)\left(\mathbf{x}^{o p t}(t+1) \mathbf{e}\right)^{-1}-\mathbf{D}\right|
$$

Finally, the question as to whether implementation of the optimal policy on the manpower system leads to retrenchment is addressed. This is done by introducing a vector $\mathbf{R}(t+1)$ as the refinement vector. The refinement vector is defined as:

$$
\mathbf{R}(t+1)=\left[\begin{array}{llll}
\sum_{j=1}^{k} p_{1 j}^{o p t} x_{1}(t) & \sum_{j=1}^{k} p_{2 j}^{o p t} x_{2}(t) & \cdots & \sum_{j=1}^{k} p_{k j}^{o p t} x_{k}(t)
\end{array}\right]-\mathbf{x}(t)
$$

The entries in $\mathbf{R}(t+1)$ are either zero or negative. This is because

$$
\sum_{j=1}^{k} p_{i j}^{o p t} \leq 1
$$

A negative entry in $\mathbf{R}(t+1)$ indicates retrenchment in the category corresponding to the position of the entry, while a zero entry implies no retrenchment. The magnitude of the entry in $\mathbf{R}(t+1)$ gives the number of staff to be retrenched. The change in the total manpower stock at the one-step period $t+1$ as a result of implementing the optimal policy is

$$
\delta \mathbf{x}(t+1)=\text { total inflow of new recruits }- \text { total outflow due to retrenchment }
$$

where $\delta \mathbf{x}(t+1)$ denotes the change in the total manpower stock at the one-step period $t+1$. Therefore 


$$
\delta \mathbf{x}(t+1)=h(t+1)+\mathbf{R}(t+1) \mathbf{e}
$$

When $\delta \mathbf{x}(t+1)>0$, the optimum policy leads to an expansion in the total manpower stock and $\delta \mathbf{x}(t+1)<0$ indicates that the optimal policy creates a contraction in the total stock. The total manpower stock is unchanged when $\delta \mathbf{x}(t+1)=0$.

\section{Application}

The utility of the model in this paper is illustrated using data on a universityfaculty setting in Nigeria. The university system in Nigeria is regulated by the National Universities Commission (NUC). The commission provides guidelines for program evaluation in the university system. Among the guidelines is the academic staff-mix by rank, which states that the existing staff structure for academic staff should closely follow the structure 20:35:45 for Professors/Readers: Senior Lecturers: Lecturer I and below (excluding the position of Graduate Assistant), respectively [25]. The study utilises data from the Faculty of Physical Sciences at the University of Benin, Nigeria [7]. The Faculty of Physical Sciences consists of five departments. We denote the departments as D1, D2, D3, D4, D5. The structure, wastage and the recruitment distribution over time for each department in the faculty as well as the initial Euclidean norms are given as follows:

D1:

$$
\mathbf{x}(t)=\left[\begin{array}{lll}
9 & 4 & 12
\end{array}\right], \mathbf{w}(t)=\left[\begin{array}{lll}
\frac{1}{43} & \frac{1}{41} & \frac{5}{57}
\end{array}\right], \mathbf{r}(t)=\left[\begin{array}{lll}
1 & 0 & 0
\end{array}\right], E_{0}=0.1226 .
$$

D2:

$$
\mathbf{x}(t)=\left[\begin{array}{lll}
16 & 9 & 2
\end{array}\right], \mathbf{w}(t)=\left[\begin{array}{ccc}
\frac{2}{109} & 0 & 0
\end{array}\right], \mathbf{r}(t)=\left[\begin{array}{lll}
1 & 0 & 0
\end{array}\right], E_{0}=0.0365
$$

D3:

$$
\begin{gathered}
\mathbf{x}(t)=\left[\begin{array}{lll}
4 & 4 & 5
\end{array}\right], \mathbf{w}(t)=\left[\begin{array}{lll}
0 & \frac{1}{25} & \frac{2}{26}
\end{array}\right], \mathbf{r}(t)=\left[\begin{array}{lll}
7 / 8 & 1 / 8 & 0
\end{array}\right] \\
E_{0}=0.0561
\end{gathered}
$$


D4:

$$
\mathbf{x}(t)=\left[\begin{array}{lll}
14 & 6 & 12
\end{array}\right], \mathbf{w}(t)=\left[\begin{array}{lll}
\frac{1}{83} & 0 & \frac{1}{34}
\end{array}\right], \mathbf{r}(t)=\left[\begin{array}{lll}
1 & 0 & 0
\end{array}\right], E_{0}=0.0572 .
$$

D5:

$$
\begin{gathered}
\mathbf{x}(t)=\left[\begin{array}{lll}
8 & 4 & 3
\end{array}\right], \mathbf{w}(t)=\left[\begin{array}{lll}
\frac{2}{41} & \frac{1}{26} & \frac{3}{13}
\end{array}\right], \mathbf{r}(t)=\left[\begin{array}{lll}
8 / 9 & 1 / 9 & 0
\end{array}\right], \\
E_{0}=0.0139
\end{gathered}
$$

The university's Annual Performance Evaluation Report (APER) form stipulates a minimum of 17 points and 20 points from publications for consideration to the rank of Senior Lecturer and Associate Professor, respectively. Apart from the points assigned to publications, there are other tasks required from the academic staff without incurring any points. Such tasks require a subjective allocation of points. Examples of such tasks are teaching and supervision of projects, external examination, administrative duties, etc. Arbitrary points are assigned to these tasks. The throughput per staff for each category is quantified by assigning some points to the task performed in each category as follows:

- Lecturer I and below (excluding the position of Graduate Assistant) - To be considered into this category, a minimum of a masters' degree is required. Apart from research, staff members in this category are to be engaged in teaching and supervision of projects at the undergraduate level. Throughput per staff in this category is allocated 5 points.

- Senior Lecturer - The throughput per staff in this category is allocated 28 points. This comprises a minimum of 17 points from publications, as well as teaching and supervision of projects at both the undergraduate and postgraduate levels (11 points).

- Professors/Associate Professor - The throughput per staff in this category is allocated 38 points. This comprises a minimum of 20 points from publications to be eligible for promotion to the rank of Associate Professor, the teaching and supervision of projects at both the undergraduate and postgraduate levels (11 points), external examination ( 2 points) and administrative duties ( 5 points).

The model proposed in this study and the model in the literature [4] are both illustrated for the case where the university management seeks to recruit 8 new academic staff into each department. At first, the optimal policy is determined using the proposed model (LPP 2) in this study.

Using the aforementioned throughput points and data, the LPP 2 model is implemented in the MATLAB environment. The implementation involves 
introducing six slack variables to augment the LPP in such fashion that the initial inverse basis matrix is

$$
\mathbf{B}_{(0)}^{-1}=\left[\begin{array}{cccccc}
1 & 0 & 0 & 0 & 0 & 0 \\
0 & 1 & 0 & 0 & 0 & 0 \\
0 & 0 & 1 & 0 & 0 & 0 \\
0 & 0 & 0 & 1 & 0 & 0 \\
0 & 0 & 0 & 0 & 1 & 0 \\
0 & 0 & 0 & 0 & 0 & 1
\end{array}\right] .
$$

The results for D1 are presented in detail and comments are provided for the others. For the instances where integer values are required, the results are rounded to the nearest whole number. For D1, the coefficient vector of the objective function is

$$
\Phi=\left[\begin{array}{lllll}
45 & 252 & 112 & 152 & 456
\end{array}\right]
$$

and the coefficient matrix of the functional constraints is

$$
\mathbf{A}=\left[\begin{array}{ccccc}
9 & 0 & 0 & 0 & 0 \\
0 & 9 & 4 & 0 & 0 \\
0 & 0 & 0 & 4 & 12 \\
1 & 1 & 0 & 0 & 0 \\
0 & 0 & 1 & 1 & 0 \\
0 & 0 & 0 & 0 & 1
\end{array}\right] .
$$

The right hand side vector is

$$
\Delta=\left[\begin{array}{llllll}
6 & 11 & 6 & 1 & 1 & 1
\end{array}\right]^{\prime}
$$

Using these matrices and vectors as inputs in the MATLAB environment, we get the following optimal solutions: 


$$
\begin{gathered}
\mathbf{B}_{(6)}^{-1}=\left[\begin{array}{cccccc}
0 & -0.1111 & 0 & 1.0000 & 0.4444 & 0 \\
1.0000 & 1.0000 & 0 & -9.0000 & -4.0000 & 0 \\
0 & 0 & 0.0833 & 0 & 0 & 0 \\
0 & 0.1111 & 0 & 0 & -0.4444 & 0 \\
0 & 0 & 0 & 0 & 1.0000 & 0 \\
0 & 0 & -0.0833 & 0 & 0 & 1.0000
\end{array}\right], \\
\mathbf{P}^{\text {opt }}=\left[\begin{array}{ccc}
0.2222 & 0.7778 & 0 \\
0 & 1.0000 & 0 \\
0 & 0 & 0.5000
\end{array}\right], \\
z 1^{\text {opt }}=546, \mathbf{x}^{\text {opt }}(t)=\left[\begin{array}{lll}
10 & 11 & 6
\end{array}\right], \mathbf{R}(t+1)=\left[\begin{array}{lll}
0 & 0 & -6
\end{array}\right],
\end{gathered}
$$

and the Euclidean norm is 0.0101 . The optimal policy is to retrench 6 staff in category 3 and that no one should be promoted in category 2, whereas about $78 \%$ of the staff in category 1 should be promoted to category 2 .

For D2, we obtain

$$
\begin{gathered}
\mathbf{P}^{\text {opt }}=\left[\begin{array}{ccc}
0.4375 & 0.5625 & 0 \\
0 & 0.3333 & 0.6667 \\
0 & 0 & 0.5000
\end{array}\right], \\
z 2^{\text {opt }}=637, \mathbf{x}^{\text {opt }}(t)=\left[\begin{array}{lll}
15 & 12 & 7
\end{array}\right], \mathbf{R}(t+1)=\left[\begin{array}{lll}
0 & 0 & -1
\end{array}\right],
\end{gathered}
$$

and the Euclidean norm is $1.2111 \times 10^{-4}$. The results show that the optimal policy is to retrench 1 staff in category 3 , while $56 \%$ and $67 \%$ of the staff in categories 1 and 2 should be promoted, respectively.

For D3,

$$
\begin{gathered}
\mathbf{P}^{\text {opt }}=\left[\begin{array}{ccc}
0.5000 & 0.5000 & 0 \\
0 & 1.0000 & 0 \\
0 & 0 & 0.8000
\end{array}\right], \\
z 3^{\text {opt }}=330, \mathbf{x}^{\text {opt }}(t)=\left[\begin{array}{lll}
9 & 7 & 4
\end{array}\right], \mathbf{R}(t+1)=\left[\begin{array}{lll}
0 & 0 & -1
\end{array}\right],
\end{gathered}
$$


and the Euclidean norm is 0 . Here the optimal staff-mix is exactly as specified by the NUC. The optimal policy is that 1 staff should be retrenched from category 3 , no staff should be promoted in category 2 and $50 \%$ of the staff in category 1 should be promoted.

For D4,

$$
\begin{gathered}
\mathbf{P}^{\text {opt }}=\left[\begin{array}{ccc}
0.4286 & 0.5714 & 0 \\
0 & 1.0000 & 0 \\
0 & 0 & 0.6667
\end{array}\right], \\
z 4^{\text {opt }}=726, \mathbf{x}^{\text {opt }}(t)=\left[\begin{array}{lll}
14 & 14 & 8
\end{array}\right], \mathbf{R}(t+1)=\left[\begin{array}{lll}
0 & 0 & -4
\end{array}\right],
\end{gathered}
$$

and the Euclidean norm is 0.0057 . For this department, 4 staff in category 3 should be retrenched. Even though no staff should be promoted in category 2, the results reveal that $57 \%$ of the staff in category 1 should be promoted.

For D5,

$$
\begin{gathered}
\mathbf{P}^{\text {opt }}=\left[\begin{array}{ccc}
0.3750 & 0.6250 & 0 \\
0 & 0.5000 & 0.5000 \\
0 & 0 & 0.6667
\end{array}\right], \\
z 5^{\text {opt }}=363, \mathbf{x}^{\text {opt }}(t)=\left[\begin{array}{lll}
10 & 8 & 4
\end{array}\right], \mathbf{R}(t+1)=\left[\begin{array}{lll}
0 & 0 & -1
\end{array}\right],
\end{gathered}
$$

and the Euclidean norm is $5.3719 \times 10^{-4}$. The optimal policy is to retrench 1 staff in category 3 and that $63 \%$ and $50 \%$ of the staff in categories 1 and 2 should be promoted, respectively.

Next the attainability problem is solved for the university-faculty using the model of Bartholomew et al. [4] and then the obtained results are compared with that obtained by using the proposed model in this paper. Using equations (8), (12) (15), the matrix $\mathbf{P}$ is computed and thereafter the discrepancy between the desired structure and the structure obtained by the model of Bartholomew et al. [4] is measured. The following results are obtained.

For D1,

$$
\mathbf{P}^{\text {Barth }}=\left[\begin{array}{ccc}
0.6101 & 0.3667 & 0 \\
0 & 0.3300 & 0.6456 \\
0 & 0 & 0.3348
\end{array}\right], E=0.0338
$$


For D2,

$$
\mathbf{P}^{\text {Barth }}=\left[\begin{array}{ccc}
0.4660 & 0.5156 & 0 \\
0 & 0.1481 & 0.8519 \\
0 & 0 & -0.3333
\end{array}\right], E=0.0021 .
$$

For D3,

$$
\mathbf{P}^{\text {Barth }}=\left[\begin{array}{ccc}
0.4934 & 0.5066 & 0 \\
0 & 0.3273 & 0.6327 \\
0 & 0 & 0.3339
\end{array}\right], E=0.0207 .
$$

For D4,

$$
\mathbf{P}^{\text {Barth }}=\left[\begin{array}{ccc}
0.6770 & 0.3109 & 0 \\
0 & 0.3015 & 0.6985 \\
0 & 0 & 0.3174
\end{array}\right], E=0.0375
$$

For D5,

$$
\mathbf{P}^{\text {Barth }}=\left[\begin{array}{ccc}
0.2675 & 0.6837 & 0 \\
0 & 0.1036 & 0.8579 \\
0 & 0 & 0.3894
\end{array}\right], E=0.0022 \text {. }
$$

Comparing these results and that obtained using the proposed model, it is easy to see that the model proposed in this study is better than that of Bartholomew et al. [4] owing to the fact that the model in this paper gives admissible solutions (unlike that of Bartholomew et al. wherein $p_{33}$ of D2 is negative) and the proposed model produces smaller discrepancies according to the Euclidean norm. Furthermore, this paper ascertains whether the optimal admissible solution obtained by the proposed model is contained in the infinitely many solutions of the model of Bartholomew et al. [4]. This is achieved by working out the product $\mathbf{q}(t) \mathbf{P}^{\text {opt }}$ and simplifying the expression $(1+\alpha) \mathbf{D}-\mathbf{q}(t)\left(\mathbf{w}^{\prime}+\mathbf{e}^{\prime} \alpha\right) \mathbf{r}$. It is found that 


$$
\mathbf{q}(t) \mathbf{P}^{o p t} \neq(1+\alpha) \mathbf{D}-\mathbf{q}(t)\left(\mathbf{w}^{\prime}+\mathbf{e}^{\prime} \alpha\right) \mathbf{r}
$$

This result leads to the conclusion that an optimal solution may not be guaranteed by the model in the literature [4]. In all, the implementation of the optimal policy leads to an expansion in the manpower stock. Comparing the norms before and after the use of the optimal promotion control strategy, it is found that there is a reduction in the Euclidean norms. Therefore, the promotion strategy proposed in this paper is capable of generating a structure which is close to the desired staffmix. The proposed model therefore is a significant contribution to the manpower planning literature on attainable structures under promotion control.

\section{Concluding remarks}

This paper presents a linear programming model for determining the one-step promotion rates that tends to equalise the attainability benchmark for a manpower system. The model characterises the evolution of structures in the system by a Markov chain. The closest rivalry to the present study is the model for promotion control proposed by Bartholomew et al. [4]. However, the findings in this study reveal that the model proposed in this paper is better than that of Bartholomew et al. [4] in the sense that: the new model gives smaller discrepancies according to the Euclidean norm, it is optimal given that it maximises the throughput, and it yields admissible solution. Our model is based on the aggregation of staff in each category of the system. Nonetheless, there are some practical issues that may limit the use of the model proposed here. These issues include, but not limited to, the psychological effect of being denied promotion, the failure of staff to meet the promotion criteria, and the yardstick to discriminate among staff to be stepped down for promotion. More so, the application of our model shows that, even though no recruitment is allowed into category 3, at least one staff in the rank of either an Associate Professor or a Professor should be retrenched. This seems reasonable as it will give room to those in the lower ranks to progress to category 3 . However, the study does not consider the subjective cost of laying-off the staff in the professorial cadre as well as the burden of severance pay to the system. Furthermore, the study utilises a simplifying assumption of equal throughput per staff per category as the performance rating per individual was not considered. In reality, some staff may be exceptional in the performance of their duties. Though these are useful areas for future research, the model proposed in this study should therefore be applied with caution. 


\section{Acknowledgement}

The author wishes to thank the Dean of the Faculty of Physical Sciences, Prof. S. E. Omosigho, and Prof. A. A. Osagiede for their encouragement and the anonymous referees for their insightful comments and suggestions which led to the revision of the earlier manuscript.

\section{References}

[1] Agnihothri, S. R., Mishra, A. K. and Simmons, D. E. (2003). Workforce crosstraining decisions in field service systems with two job types. The Journal of the Operational Research Society, 54(4), 410-418.

[2] Anderson, E. G. (2001). The nonstationary staff-planning problem with business cycle and learning effects. Management Science, 47(6), 817-832.

[3] Arlotto, A., Chock, S. E. and Gans, N. (2014). Optimal hiring and retention policies for heterogeneous workers who learn. Management Science, 60(1), 110-129.

[4] Bartholomew, D. J., Forbes, A. F. and McClean, S.I. (1991). Statistical Techniques for Manpower Planning (2nd ed.). Chichester: John Wiley \& Sons.

[5] Bordoloi, S. K. (2006). A control rule for recruitment planning in engineering consultancy. Journal of Productivity Analysis, 26(2), 147-163.

[6] Davis, B. A., Heathcote, C. R. and O'Neill, T. J. (2002). Estimating and interpolating Markov chain from aggregate data. Biometrika, 89(1), 95-110.

[7] Ekhosuehi, V. U. (2013). Evaluation of career patterns of academic staff in a faculty in the University of Benin, Nigeria. Ife Journal of Science, 15(1), 8191.

[8] Ekhosuehi, V. U. and Osagiede, A. A. (2013). A proposed Markov model for predicting the structure of a multi-echelon educational system in Nigeria. Monografias Matematicas Garcia de Galdeano, 38, 65-74.

[9] Ekhosuehi, V. U., Osagiede, A. A. and Iguodala, W. A. (2015). A procedure for distributing recruits in manpower systems. Yugoslav Journal of Operations Research, 25(3), 441-452. DOI: 10.2298/YJOR131219031E.

[10] Ferreira, M. A. R. and Suchard, M. A. (2008). Bayesian analysis of elapsed times in continuous-time Markov chains. The Canadian Journal of Statistics, 36(3) 355-368.

[11] Fry, M. J., Magazine, M. J. and Rao, U. S. (2006). Firefighter staffing including temporary absences and wastage. Operations Research, 54(2), 353-365. 
[12] Gans, N. and Zhou, Y.-P. (2002). Managing learning and turnover in employee staffing. Operations Research, 50(6), 991-1006.

[13] Guo, X. and Hernandez-Lerma, O. (2003). Continuous-time controlled Markov chains. The Annals of Applied Probability, 13(1), 363-388.

[14] Harper, P. R., Powell, N. H. and Williams, J. E. (2010). Modelling the size and skill-mix of hospital nursing teams. The Journal of the Operational Research Society, 61(5), 768-779.

[15] Hillier, F. S. and Lieberman, G. J. (2005). Introduction to Operations Research (8th ed.). New York: McGraw-Hill.

[16] Ibe, O. C. (2009). Markov Processes for Stochastic Modelling. Burlington: Elsevier Academic Press.

[17] Kipouridis, I. and Tsaklidis, G. (2001). The size order of the state vector of discrete-time homogeneous Markov systems. Journal of Applied Probability, 38(2) 357-368.

[18] Mitrophanov, A. Y. (2003). Stability and exponential convergence of continuous-time Markov chains. Journal of Applied Probability, 40(4), 970-979.

[19] Nicholls, M. G. (2007). Assessing the progress and the underlying nature of the flows of doctoral and master degree candidates using absorbing Markov chains. Higher Education, 53(6) 769-790.

[20] Nicholls, M. G. (2009). The use of Markov models as an aid to the evaluation, planning and benchmarking of doctoral programs. Journal of the Operational Research Society, 60, 1183-1190.

[21] Nilakantan, K. (2013). Estimation of career progression and growth properties in Markov manpower systems with a competitive climate. International Journal of Business and Systems Research, 7(3), 292-316.

[22] Nilakantan, K. (2014). Evaluation of staffing policies in Markov manpower systems and their extension to organizations with outsource personnel. Journal of the Operational Research Society. DOI: 10.1057/jors.2014.82.

[23] Oliva, R. and Sterman, J. D. (2001). Cutting corners and working overtime: quality erosion in the service industry. Management Science, 47(7), 894-914.

[24] Osagiede, A. A. and Ekhosuehi, V. U. (2015). Finding a continuous-time Markov chain via sparse stochastic matrices in manpower systems. Journal of the Nigerian Mathematical Society, 34, 94-105.

DOI: $10.1016 /$ j.jnnms.2014.10.004.

[25] Osasona, O. (2012). Tools for academic planning. In Uvah, I. I. (Editor). Practical Guide on Academic Planning in Nigerian Universities: A Compendium of Academic Planning Tools (pp. 62-105). 
[26] Ploskas, N. and Samaras, N. (2014). Pivoting rules for the revised simplex algorithm. Yugoslav Journal of Operations Research, 24(3), 321-332. DOI: 10.2298/ YJOR140228016P.

[27] Pollett, P. K. (2001). Similar Markov chains. The Journal of Applied Probability, 38(A), 53-65.

[28] Pritchard, G. and Scott, D. J. (2001). Empirical convergence rates for continuous-time Markov chains. Journal of Applied Probability, 38(1), 262269.

[29] Udom, A. U. (2013). A Markov decision process approach to optimal control of a multi-level hierarchical manpower system. CBN Journal of Applied Statistics, 4(2), 31-49.

[30] Udom, A. U. (2014). Optimal controllability of manpower system with linear quadratic performance index. Brazilian Journal of Probability and Statistics, 28(2) 151-166. DOI: 10.1214/12-BJPS195.

[31] Yap, V. B. (2009). Similar states in continuous-time Markov chains. Journal of Applied Probability, 46(2), 497-506.

[32] Yin, G. and Zhang, H. (2005). Two-time-scale Markov chains and applications to quasi-birth-death queues. SIAM Journal on Applied Mathematics, 65(2), 567-586.

[33] Zhu, X. and Sherali, H.D. (2009). Two-stage workforce planning under demand fluctuations and uncertainty. The Journal of Operational Research Society, 60(1) 94-103. 